\title{
Highly sensitive pressure sensors employing 3C-SiC nanowires fabricated on a free standing structure
}

Hoang-Phuong Phan ${ }^{\mathrm{a}, \mathrm{b}}$, Karen M. Dowling ${ }^{\mathrm{b}}$, Tuan Khoa Nguyen ${ }^{\mathrm{a}}$, Toan Dinh ${ }^{\mathrm{a}}$, Debbie G. Senesky ${ }^{\mathrm{b}, \mathrm{c}}$, Takahiro Namazu ${ }^{\mathrm{d}}$, Dzung Viet Dao ${ }^{\mathrm{a}, \mathrm{e}}$, Nam-Trung Nguyen ${ }^{\mathrm{a}}$

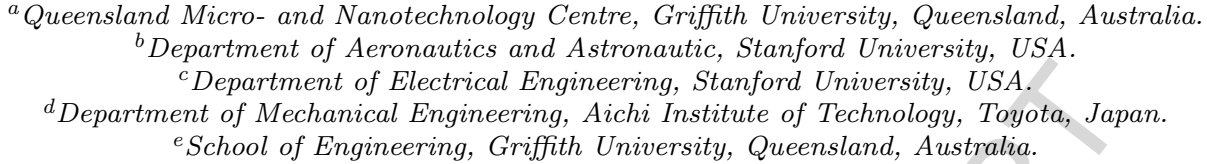

\begin{abstract}
This paper presents highly sensitive pressure sensors using piezoresistive nanowires. Our approach is based on nanowires locally fabricated on free standing structures with a high strain concentration. This strain concentration phenomenon amplifies the strain induced into nano-scaled sensing elements while the bulk materials are still at small strain regime, therefore enhancing the sensitivity of the sensors. For proof of concept, we utilized SiC nanowire fabricated using focused ion beam from an epitaxially grown thin film. Experimental results show significant 3-fold enhancement in the sensitivity in comparison to conventional structures, which is in good agreement with analytical modeling and numerical simulation. The proposed design shows potential for the development of miniaturized highly sensitive but robust nano mechanical-sensors.
\end{abstract}

Keywords: Piezoresistance, Nanowires, Pressure sensors, SiC

\section{Introduction}

Nanowires have been considered as an important building block for the next generation of Nano Electro Mechanical Systems (NEMS) sensors [1. The use of nanowires provides numerous advantages over conventional bulk materials including device miniaturization, high sensitivity, and superior optical properties [2, 3, 4, 5]. For instance, by assembling multiple silicon nanowires on a single chip, the Lieber group has developed miniaturized computers which can perform the basic logic states including AND, OR, NOT, and NAND with a relatively low gate voltage [6]. In addition, the sub-wavelength diameter and the capability of tuning energy gaps also make nanowires a promising platform for the generation, detection and modulation of light. As such, Liu et al. reported wavelength tunable lasers by modulating the alloy composition in $\mathrm{Zn}_{x} \mathrm{Cd}_{1-x} \mathrm{~S}$ nanowires [7. Furthermore, the nanoscale

\footnotetext{
* Corresponding author

Email address: hoangphuong.phan@griffithuni.edu.au (Hoang-Phuong Phan)
} 
dimensions allow charge carriers to be exposed on the surface of nanowires, making them sensitive to stimuli from surrounding environments. A well-known exemplar application of this property is $\mathrm{Si}$ nanowires for label-free biosensors, which exhibit fast response and large signal-to-noise ratios owing to their large surface area 8 .

Nanowires have also been employed in highly sensitive mechanical sensors such as strain and pressure sensors. The use of a nanowire as the channel of a field effect transistor (FET) could enhance strain-sensitivity by modulating the top gate [9, 10, 11]. A number of studies have been conducted to improve the sensitivity of MEMS pressure and strain sensors using this phenomenon in nanowire FETs [12, 13, 14].

In addition to their exhibiting excellent electrical and optical properties, the nanoscales nanowires have also been proven to be much stronger than bulk materials due to the smaller defect concentrations [15]. In our previous studies, we have demonstrated that Si nanowires can withstand strain of at least $2.5 \%$, whereas bulk Si generally exhibits brittleness at less than $1 \%$ strain [16. The Cui group has demonstrated the use of Si nanowires forests instead of bulk electrodes in lithium batteries, showing significant enhancement in long-term stability of nanowires over bulk materials due to their tolerance to tensile strain during charging/discharging processes [17. The strong mechanical properties of nanowires imply that in mechanical sensing applications, it is desirable to concentrate mechanical impact on nano structures, while maintaining lower stress/strain on micro structures so that the sensors could offer higher sensitivity and improved robustness [18].

In the present work we propose an effective approach to develop highly sensitive pressure sensors employing nanowires fabricated at the center of a dogbone-like structure. The key concept of our design relies on the strain concentration phenomenon to maximize mechanical stimuli applied to nanowires, enhancing the sensitivity of the sensors. Experimental results show an approximately 3 -fold improvement in the sensitivity of $\mathrm{SiC}$ nanowire pressure sensors in comparison to bulk-material-based sensors of the same dimensions.

\section{Results and discussion}

\subsection{Concept of nanowire based pressure sensors}

Figure 1 shows the concept of a nanowires pressure sensors, in which the nanowires is fabricated at the center of a free-standing micro bridge. The bridge is suspended on a thick membrane, which is used to transfer the strain from external air-pressure to the sensing element. The design was inspired by the dog-bone structure which is widely employed to test the tensile strength of materials. That is, when a sufficiently large mechanical load is applied to dogbone-structured materials, cracks always occur at the narrow section of the dog-bone due to the stress concentration phenomenon. Similarly, in 
(a) Concept of pressure sensors

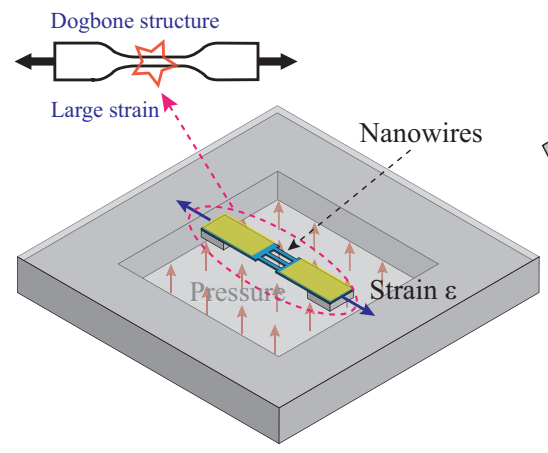

(b) Principle

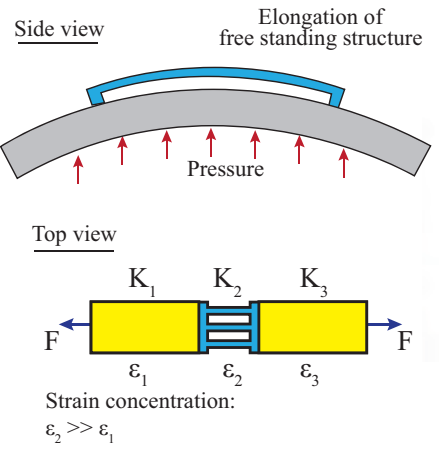

(c) Numerical estimation

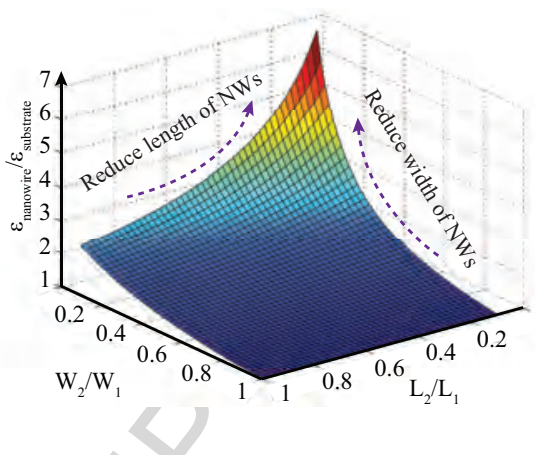

Figure 1: Concept of the pressure sensors. (a) Nanowire fabricated at the center of a free standing micro bridge; (b) Schematic sketch of strain induced into the free standing nanowire and micro bridge under pressure; (c) Estimation of the strain amplification rate when varying the dimension of nanowire arrays.

our structure, upon applying pressure the upper surface of the membrane will be deflected outward, and a large elongation is expected to be observed in the narrowed nanowires areas. This assumption can be quantitatively explained as follows.

Under the applied pressure $p$, the strain induced into the center point of the top surface of the membrane $\left(\varepsilon_{\text {sub }}\right)$ is given by [19]:

$$
\varepsilon_{s u b}=\frac{0.1386 \times p \times b^{2}}{E \times t^{2}}
$$

where $b$ and $t$ are the width and the thickness of the membrane; and $E$ is the Young's modulus of the substrate.

Since the stiffness of the suspended structure (including the microscaled parts and the nanowires located at the center) is much smaller that the stiffness of the membrane, upon applying pressure, this free standing structure will be elongated following the deflection of the membrane. The change in the length of the suspended bridge is:

$$
\Delta L=L \times \varepsilon_{s u b}=L_{1} \times \varepsilon_{1}+L_{2} \times \varepsilon_{2}+L_{3} \times \varepsilon_{3}
$$

where $\varepsilon_{i}$ is strain induced into each component of the free standing dog-bone structure. For a symmetrical design, $\varepsilon_{1}$ and $\varepsilon_{3}$ are equivalent and $\Delta L=2 \times L_{1} \times \varepsilon_{1}+L_{2} \times \varepsilon_{2}$.

Due to the force equilibrium in the suspended structure, the following equation is established following Hooke's law:

$$
F=K_{1} \times \varepsilon_{1}=K_{2} \times \varepsilon_{2}=K_{3} \times \varepsilon_{3}
$$

where $K_{1}$ is the stiffness of the microscaled frame, and $K_{2}$ is the stiffness of the nanowires. From Eq. 3 the ratio of the strains induced into nanowires and micro frame is:

$$
\varepsilon_{2}=\left(K_{1} / K_{2}\right) \varepsilon_{1}
$$


Substituting Eq. 4 into Eq. 2, the strain induced into the nanowires is:

$$
\varepsilon_{2}=\frac{L}{2 \frac{K_{2}}{K_{1}} L_{1}+L_{2}} \times \varepsilon_{\text {sub }}=\frac{1}{1-2\left(1-\frac{K_{2}}{K_{1}}\right) \frac{L_{1}}{L}} \times \varepsilon_{\text {sub }}
$$

It is evident from Eq. 5 that the softer $\left(K_{2}<<K_{1}\right)$ and shorter $\left(L_{2}<<L_{1}\right)$ the nanowires are, the larger is the strain concentrated in the nanowires (i.e. $\varepsilon_{2}>>\varepsilon_{s u b}$ ), Fig. 1.(c). In other words, the strain induced into the sensing element (i.e. the nanowires), can be significantly amplified from the applied strain $(\varepsilon)$ by:

(i) reducing the width of the nanowires (e.g. reduce the ratio of $K_{2} / K_{1} \approx W_{2} / W_{1}$ )

(ii) shortening the length of the nanowires in comparison to the microscale parts (e.g. increasing the ratio of $\left.L_{1} / L\right)$

It should be noted that the strain applied to the bulk material $\left(\varepsilon_{\text {sub }}\right)$ remains small, allowing the sensors to function in a wider range of pressures while still offering the high sensitivity.

\subsection{Characterization of nanowires based pressure sensors}

To demonstrate the sensitivity enhancement in the proposed structure, we developed piezoresistive $\mathrm{SiC}$ nanowires on Si-membrane pressure sensors, Fig. 2. Cubic SiC was selected in this work due to the increasing demand for the development of wide-band-gap based mechanical sensors for applications in harsh environments including high temperatures and high pressures [20, 21, 22, 23. P-type SiC films with thickness of $300 \mathrm{~nm}$, was deposited on (100) Si using chemical vapor deposition at $1000^{\circ} \mathrm{C}$. A detailed description of the deposition process and characterization of the as-grown film has been reported in our previous studies 24]. The $\mathrm{SiC}$ layer was then patterned using Inductive Coupled Plasma Etching through to the Si layer with an etching rate of $100 \mathrm{~nm} / \mathrm{min}$. Subsequently, $\mathrm{Al}$ was deposited and then wet-etched to form electrode pads. SiC micro bridges with dimension of $200 \mu \mathrm{m}$ in length, $8 \mu \mathrm{m}$ in width and $300 \mathrm{~nm}$ in thickness were released from the substrate by isotropic etching of Si using $\mathrm{XeF}_{2}$ as the active gas [25].

Subsequently, arrays of $\mathrm{SiC}$ nanowires were fabricated using focused ion beam with $\mathrm{Ga}^{+}$ions [26. 27]. Finally, the Si membrane was formed by etching the Si substrates from the backside. For simplicity, a large scale Si membrane with dimension of $5 \mathrm{~mm} \times 5 \mathrm{~mm} \times 150 \mu \mathrm{m}$ was fabricated using a UV laser with an etching rate of $60 \mu \mathrm{m} / \mathrm{min}$ [28. For comparison, a free-standing micro SiC resistor on Si-membrane pressure sensor was also fabricated. It should also be noted that, since the sensing element was p-type cubic $\mathrm{SiC}$, the suspended bridge and the nanowires were aligned in [110] orientation to provide the highest sensitivity [29].

Figure 3(a) shows an array of 3C-SiC nanowires fabricated at the center of a suspended $200 \mu \mathrm{m}-$ length bridge. The SEM image of the nanowires taken from a $45^{\circ}$-tilted-view shows that the Si layer underneath was removed by the isotropic dry etching. The average dimension of each nanowire was 
measured at $5 \mu \mathrm{m} \times 430 \mathrm{~nm} \times 300 \mathrm{~nm}$, Fig. 3(c). Figure 3(d) shows an SEM image of the back side of the Si membranes, with an etching-depth of approximately $500 \mu \mathrm{m}$ measured using Dektak ${ }^{\mathrm{TM}} 150$ Surface Profiler (Inset). The roughness of the laser-engraved Si surface was found to be approximately $20 \mu \mathrm{m}$, Fig. 3(e). The SEM image of the SiC micro resistor used for comparison is shown in Fig. 3(f). The dimension of the $\mathrm{Si}$ membrane in the $\mathrm{SiC}$ microresistor based pressure sensor were similar to that of the nanowire based sensors $(5 \mathrm{~mm} \times 5 \mathrm{~mm} \times 150 \mu \mathrm{m})$.

Figure 4 shows the current-voltage (I-V) characteristics of $\mathrm{SiC}$ micro resistors and $\mathrm{SiC}$ nanowires with an almost linear relationship in the $\mathrm{I}-\mathrm{V}$, indicating a Ohmic contact between $\mathrm{Al}$ and $\mathrm{SiC}$ films. Furthermore, from the geometry of the $\mathrm{SiC}$ nanowires and the $\mathrm{SiC}$ micro resistors, the resistivity of the nanowires and the bulk material were found to be almost the same $(4.2 \mathrm{k} \Omega / \square)$, indicating that the FIB process did not significantly affect the electrical properties of the nanowires.

We then investigated the magnitude of the piezoresistive effect in non-suspended micro-sized $\mathrm{SiC}$ and nanowire $\mathrm{SiC}$ resistors. Non-suspended $\mathrm{SiC}$ micro resistors and $\mathrm{SiC}$ nanowires similar to that of the pressure sensors were formed on a Si beam with dimensions of $40 \mathrm{~mm} \times 8 \mathrm{~mm} \times 650 \mu \mathrm{m}$, from the same SiC-on-Si wafer (Fig. 5 inset). The bending method was utilized to apply uniaxial strain to the $\mathrm{SiC}$ micro resistor and nanowires, where the Si beam was fixed at one end using a metal clamp (See the supplementary information). Figure 5 shows the relative resistance change of the $\mathrm{SiC}$ micro resistors and nanowires under strain varying from 0 to $350 \mathrm{ppm}$. A linear relationship between resistance change and applied strain was observed in both bulk materials and nanowires. The gauge factors $(G F=(\Delta R / R) / \varepsilon)$ of the nanowires was found to be approximately 33, which is in the same range of that of bulk materials $(\mathrm{GF} \approx 30)$. These results are reasonable as the dimension of the nanowires is well above the the quantum-effect-length.

The $\mathrm{SiC}$ nanowire and micro-sized pressure sensors were then mounted on an acrylic holder with

(1)

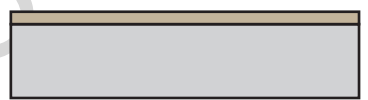

(3)

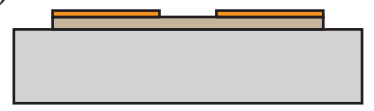

(5)
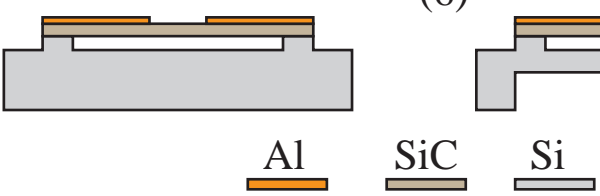

Figure 2: Fabrication process of the SiC nanowires pressure sensor
(2)

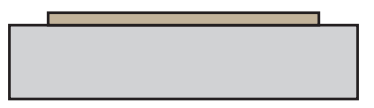

(4)

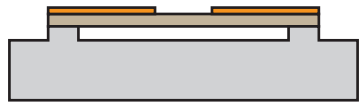

(6)

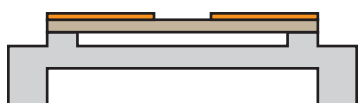

\section{$\mathrm{Si}$}



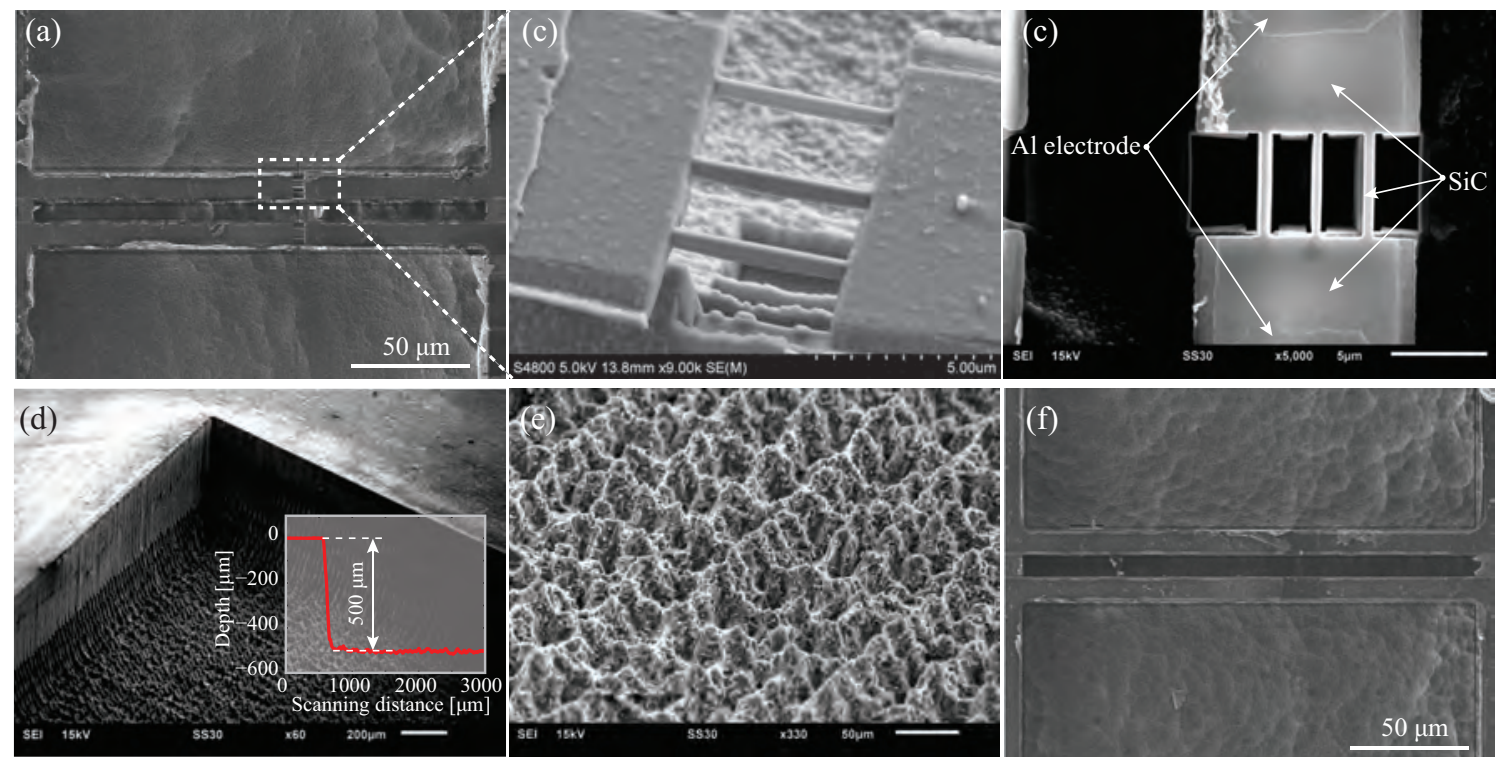

Figure 3: Photograph of fabricated devices. (a) SiC nanowires fabricated at the center of a suspended bridge; (b) Zoomin SEM image of an SiC nanowires; (c) SEM image of the nanowire array from the top view, showing the nanowires are connected to two serial micro resistors; (d)SEM image of back side Si membrane; (e) Surface roughness of Si on the backside after laser engraving; (f) A pressure sensor with suspended $\mathrm{SiC}$ microresistors fabricated on a Si membrane for comparison to nanowire based sensor.

a through hole at the center using UV gel as shown in Fig6(a). The holder was then fixed onto an enclosed metal chamber. Air pressure was applied to the backside of the Si membrane using a pressure controller (Eveflow ${ }^{\mathrm{TM}}$ ), Fig. 6(b). Finite element analysis using Comsol Multiphysics ${ }^{\mathrm{TM}}{ }_{\mathrm{was}}$

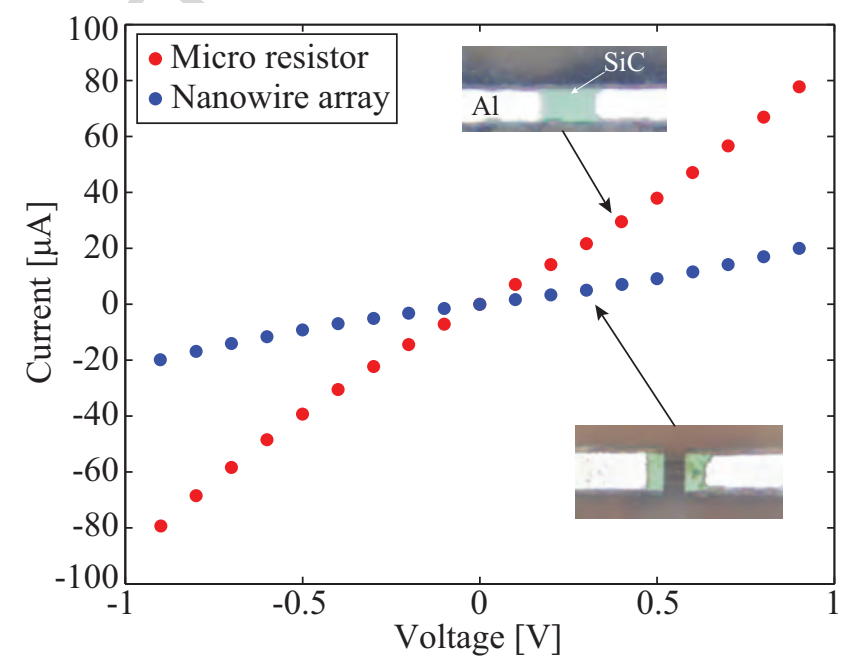

Figure 4: Current-voltage characteristics of $\mathrm{SiC}$ microresistors and nanowires by sweeping applied voltage from $-1 \mathrm{~V}$ to $1 \mathrm{~V}$. 
employed to estimate the strain induced into the $\mathrm{SiC}$ nanowires and the suspended micro bridge. Accordingly, the strain induced into the as-fabricated nanowires was derived to be approximately 5 times larger than that of the microresistors, Fig. 6(c). Using the gauge factor of the nanowires and microresistors obtained from Fig. 5, the strain amplification in the sensing element was expected to result in enhancement in the relative resistance change of the sensors by 3.5 folds in comparison to micro SiC resistors.

A pressure of 500 mbar was then cyclically applied with an interval of $8 \mathrm{~s}$ for each pressurizing cycle. The resistances of $\mathrm{SiC}$ nanowires and $\mathrm{SiC}$ micro resistors were monitored and recorded using an Agilent ${ }^{\mathrm{TM}}$ B1500A Semiconductor Device Parameter Analyzer where the applied voltage was kept at $0.5 \mathrm{~V}$. Figure $6(\mathrm{~d})$ shows the response of the sensors (the change in current) subjected to cyclic square-pressure of 500 mbar. Both SiC nanowires and microresistor-based sensors showed excellent repeatability of the resistance change over several testing cycles. Evidently, the response of the nanowires pressure sensors was approximately 3 times larger than that of the pressure sensors using micro-sized $\mathrm{SiC}$. The enhancement in the sensitivity of the as-fabricated sensors was in good agreement with the simulation result mentioned above. The difference between the experimental and simulation data could result from the surface roughness of the Si membrane due to laser engraving (Fig. 4(e)) or the sensor alignment error. It should also be pointed out that, in out experiments, for the shake of simplicity, the output of the sensors was monitored using the Device Parameter Analyzer, where a small change in the current can be detected after subtracting the initial current [30, 31, 32. However, in practical applications, the use of Wheatstone bridge combined with an Op-amp to eliminate the offset the voltage and amplify the output is recommended. The data recording method using this circuit is described in

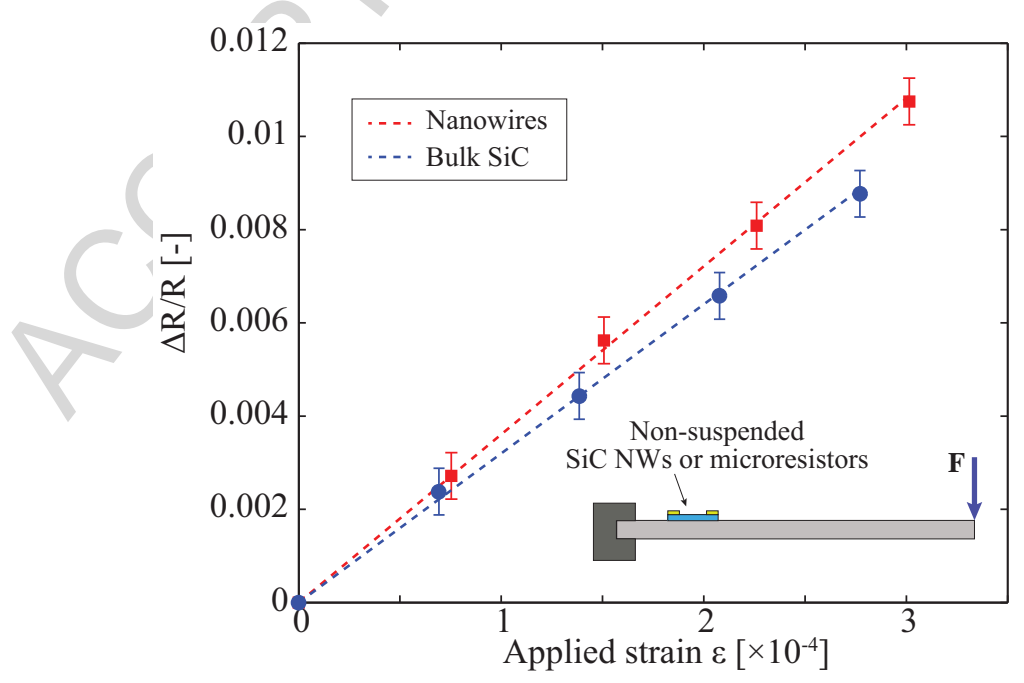

Figure 5: Comparison of the piezoresistive effect in non-suspended SiC nanowires and microresistors. 
(a)
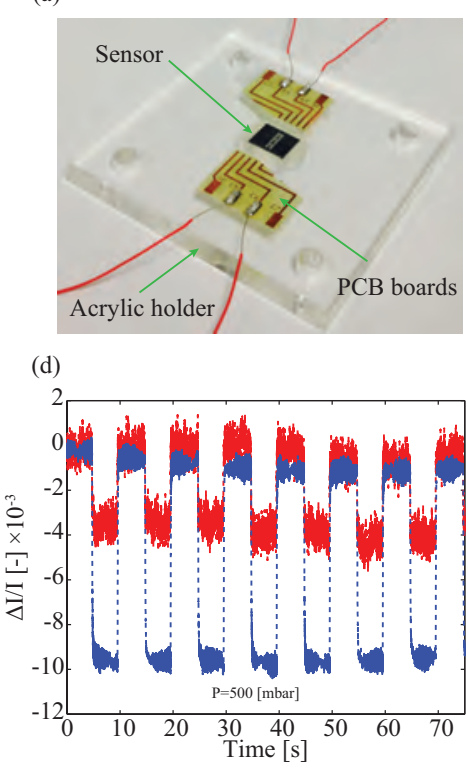

(b)

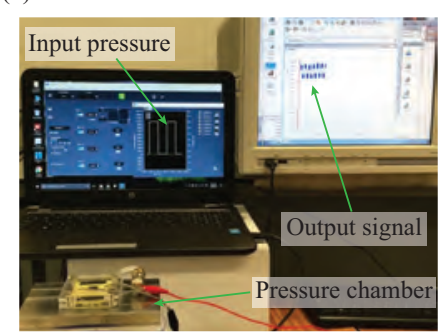

(e)

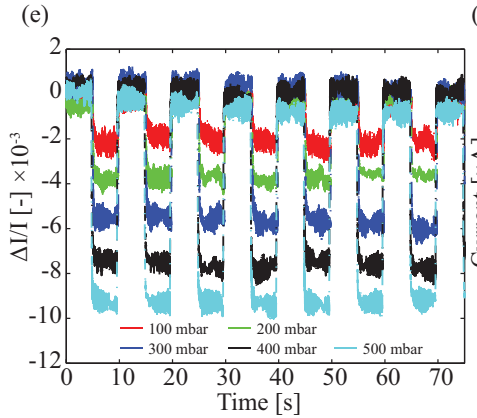

(c)

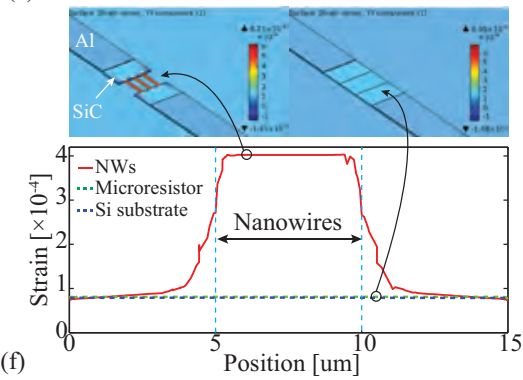

(f)

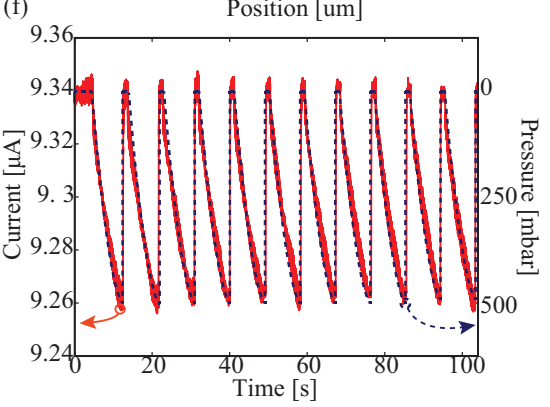

Figure 6: Characterization of SiC pressure sensors. (a) Photograph of SiC nanowire pressure sensor mounted on an acrylic holder; (b) Experimental setup of SiC nanowire pressure sensors; (c) Simulation of strain induced into the nanowires and microscaled frames; (d) A 3-fold increase in the sensitivity of nanowire sensors (blue) in comparison to SiC micro resistors (red); (e) The output of $\mathrm{SiC}$ nanowire sensor under different input-pressures. (f) Response of the nanowire pressure sensors under ramp-up pressures from 0 to 500 mbar.

the supplementary information.

The nanowire-based sensors were then subjected to different applied pressure, showing that the current change increased linearly with increasing pressures, Fig. 6(e). The output of the nanowire pressure sensors against ramped-up pressure input (8 seconds/cycle) is shown in Fig 6 ff), indicating fast time response and good repeatability with a variation of less than $3 \%$ between cycles. The sensor did not show any significant degradation after subjecting to 1,000 pressurizing-depressurizing cycles.

\section{Conclusion}

This work reports a novel structure for the development of highly sensitive pressure sensors using nanowires. The proposed concept includes a nanowire array locally fabricated at the center of a free standing micro structure on a pressure-membrane. A proof of concept pressure sensor was developed, showing approximately 3 -fold enhancement in the sensitivity in comparison to the conventional design. The proposed structure can be optimized to obtain higher sensitivity-enhancement in the future. 


\section{Acknowledgments}

This work was partially funded by the linkage grants LP150100153 and LP160101553 from the Australian Research Council (ARC). This work was performed in part at the Queensland node of the Australian National Fabrication Facility, a company established under the National Collaborative Research Infrastructure Strategy to provide nano and micro-fabrication facilities for Australia's researchers. H.P. Phan acknowledges research grants from the Australian Nanotechnology Network Overseas Travel Fellowship and the Griffith University Postdoctoral Fellowship (GUPF).

\section{Reference}

[1] A. Zhang, G. Zheng, and C. Lieber, Nanowires: building blocks for nanoscience and nanotechnology, Springer, 2017.

[2] Javey, A., Nam, S., Friedman, R.S., Yan, H. and Lieber, C.M., Layer-by-layer assembly of nanowires for three-dimensional, multifunctional electronics. Nano letters, 7(3), 773-777, 2007.

[3] Gong, S., Schwalb, W., Wang, Y., Chen, Y., Tang, Y., Si, J., Shirinzadeh, B. and Cheng, W., A wearable and highly sensitive pressure sensor with ultrathin gold nanowires. Nature communications, 5, p.3132, 2014.

[4] Eaton, S.W., Lai, M., Gibson, N.A., Wong, A.B., Dou, L., Ma, J., Wang, L.W., Leone, S.R. and Yang, P., Lasing in robust cesium lead halide perovskite nanowires. Proceedings of the National Academy of Sciences, 113(8), pp.1993-1998, 2016.

[5] Dinh, T., Phan, H.P., Kozeki, T., Qamar, A., Fujii, T., Namazu, T., Nguyen, N.T. and Dao, D.V., High thermosensitivity of silicon nanowires induced by amorphization. Materials Letters, 177, pp.80-84, 2016.

[6] Huang, Y., Duan, X., Cui, Y., Lauhon, L.J., Kim, K.H. and Lieber, C.M., Logic gates and computation from assembled nanowire building blocks. Science, 294(5545), pp.1313-1317, 2001

[7] Liu, Y., Zapien, J.A., Shan, Y.Y., Geng, C.Y., Lee, C.S. and Lee, S.T., 2005. WavelengthControlled Lasing in ZnxCd1xS SingleCrystal Nanoribbons. Advanced Materials, 17(11), pp.1372-1377.

[8] Wang, Y., Wang, T., Da, P., Xu, M., Wu, H. and Zheng, G., Silicon nanowires for biosensing, energy storage, and conversion. Advanced materials, 25(37), pp.5177-5195, 2013

[9] He, R. and Yang, P., 2006. Giant piezoresistance effect in silicon nanowires. Nature nanotechnology, $1(1)$, p.42. 
[10] Neuzil, P., Wong, C.C. and Reboud, J., 2010. Electrically controlled giant piezoresistance in silicon nanowires. Nano letters, 10(4), pp.1248-1252.

[11] Wang, Z.L., 2010. Piezopotential gated nanowire devices: Piezotronics and piezo-phototronics. Nano Today, 5(6), pp.540-552.

[12] He, R., Feng, X.L., Roukes, M.L. and Yang, P., 2008. Self-transducing silicon nanowire electromechanical systems at room temperature. Nano letters, 8(6), pp.1756-1761.

[13] Mile, E., Jourdan, G., Bargatin, I., Labarthe, S., Marcoux, C., Andreucci, P., Hentz, S., Kharrat, C., Colinet, E. and Duraffourg, L., 2010. In-plane nanoelectromechanical resonators based on silicon nanowire piezoresistive detection. Nanotechnology, 21(16), p.165504.

[14] Zhao, Z., Pu, X., Han, C., Du, C., Li, L., Jiang, C., Hu, W. and Wang, Z.L., 2015. Piezotronic effect in polarity-controlled GaN nanowires. ACS nano, 9(8), pp.8578-8583.

[15] An, B.W., Gwak, E.J., Kim, K., Kim, Y.C., Jang, J., Kim, J.Y. and Park, J.U., Stretchable, transparent electrodes as wearable heaters using nanotrough networks of metallic glasses with superior mechanical properties and thermal stability. Nano letters, 16(1), pp.471-478, 2015

[16] H.-P. Phan, T. Kozeki, T. Dinh, T. Fujii, A. Qamar, Y. Zhu, T. Namazu, N.-T. Nguyen, and D. V. Dao, Piezoresistive effect of p-type silicon nanowires fabricated by a top-down process using FIB implantation and wet etching. RSC Advances, 5(100), pp.82121-82126, 2015.

[17] Liu, N., Lu, Z., Zhao, J., McDowell, M.T., Lee, H.W., Zhao, W. and Cui, Y., A pomegranateinspired nanoscale design for large-volume-change lithium battery anodes. Nature nanotechnology, 9(3), p.187, 2014.

[18] Xiao, X., Yuan, L., Zhong, J., Ding, T., Liu, Y., Cai, Z., Rong, Y., Han, H., Zhou, J. and Wang, Z.L., HighStrain Sensors Based on ZnO Nanowire/Polystyrene Hybridized Flexible Films. Advanced materials, 23(45), pp.5440-5444, 2011

[19] Zribi, A. and Fortin, J. eds., 2009. Functional thin films and nanostructures for sensors: synthesis, physics and applications. Springer Science \& Business Media.

[20] Dao, D.V., Phan, H.P., Qamar, A. and Dinh, T., 2016. Piezoresistive effect of p-type single crystalline 3CSiC on (111) plane. RSC Advances, 6(26), pp.21302-21307.

[21] Yu, H., Zhu, J., Yang, L., Dai, B., Baraban, L., Cuniberti, G. and Han, J., 2015. Superhydrophobic carbon nanotube/silicon carbide nanowire nanocomposites. Materials \& Design, 87, pp.198-204. 
[22] Chapin, C.A., Miller, R.A., Dowling, K.M., Chen, R. and Senesky, D.G., 2017. InAlN/GaN high electron mobility micro-pressure sensors for high-temperature environments. Sensors and Actuators A: Physical, 263, pp.216-223.

[23] Colston, G., Rhead, S.D., Shah, V.A., Newell, O.J., Dolbnya, I.P., Leadley, D.R. and Myronov, M., 2016. Mapping the strain and tilt of a suspended 3C-SiC membrane through micro X-ray diffraction. Materials \& Design, 103, pp.244-248.

[24] Phan, H.P., Cheng, H.H., Dinh, T., Wood, B., Nguyen, T.K., Mu, F., Kamble, H., Vadivelu, R., Walker, G., Hold, L. and Iacopi, A., 2017. Single-Crystalline 3C-SiC anodically Bonded onto Glass: An Excellent Platform for High-Temperature Electronics and Bioapplications. ACS applied materials \& interfaces, 9(33), pp.27365-27371.

[25] Phan, H.P., Dinh, T., Kozeki, T., Qamar, A., Namazu, T., Dimitrijev, S., Nguyen, N.T. and Dao, D.V., 2016. Piezoresistive effect in p-type 3C-SiC at high temperatures characterized using Joule heating. Scientific reports, 6, p.28499.

[26] Kozeki, T., Phan, H.P., Dao, D.V., Inoue, S. and Namazu, T., 2016. Influence of gallium ion beam acceleration voltage on the bend angle of amorphous silicon cantilevers. Japanese Journal of Applied Physics, 55(6S1), p.06GL02.

[27] Foisal, A.R.M., Phan, H.P., Kozeki, T., Dinh, T., Tuan, K.N., Qamar, A., Lobino, M., Namazu, T. and Dao, D.V., 2016. 3CSiC on glass: an ideal platform for temperature sensors under visible light illumination. RSC Advances, 6(90), pp.87124-87127.

[28] Dowling, K.M., So, H., Toor, A., Chapin, C.A. and Senesky, D.G., 2017. Lithography-free microfabrication of AlGaN/GaN 2DEG strain sensors using laser ablation and direct wire bonding. Microelectronic Engineering, 173, pp.54-57.

[29] Phan, H.P., Viet Dao, D., Tanner, P., Wang, L., Nguyen, N.T., Zhu, Y. and Dimitrijev, S., 2014. Fundamental piezoresistive coefficients of p-type single crystalline 3C-SiC. Applied Physics Letters, 104(11), p.111905.

[30] Haick, H., Shuster, G., Segev-bar, M., Kloper, V. and Gliksman, S., Platform unit for combined sensing of pressure, temperature and humidity. U.S. Patent, 2017, 9625341.

[31] Park, S., Wang, G., Cho, B., Kim, Y., Song, S., Ji, Y., Yoon, M.H. and Lee, T., Flexible molecularscale electronic devices. Nature Nanotech., 7(7), p.438, 2012. 
[32] M.-D. Nguyen, H.-P. Phan, K. Matsumoto, and I. Shimoyama, A sensitive liquid-cantilever diaphragm for pressure sensor. In Proc. IEEE Int. Conf. Micro Electro Mechanical Systems (MEMS), pp. 617-620, 2013.

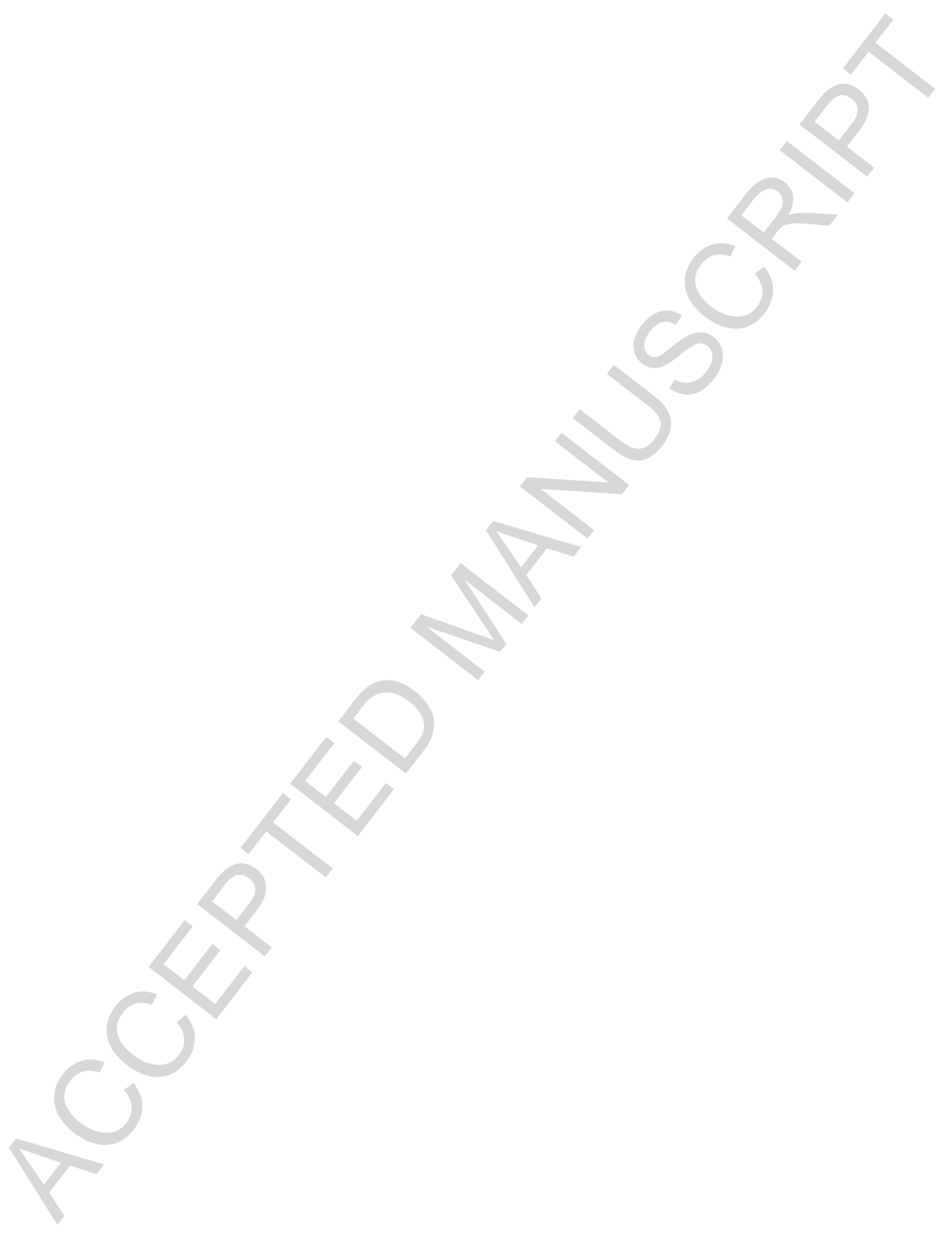




\section{Graphical abstract}
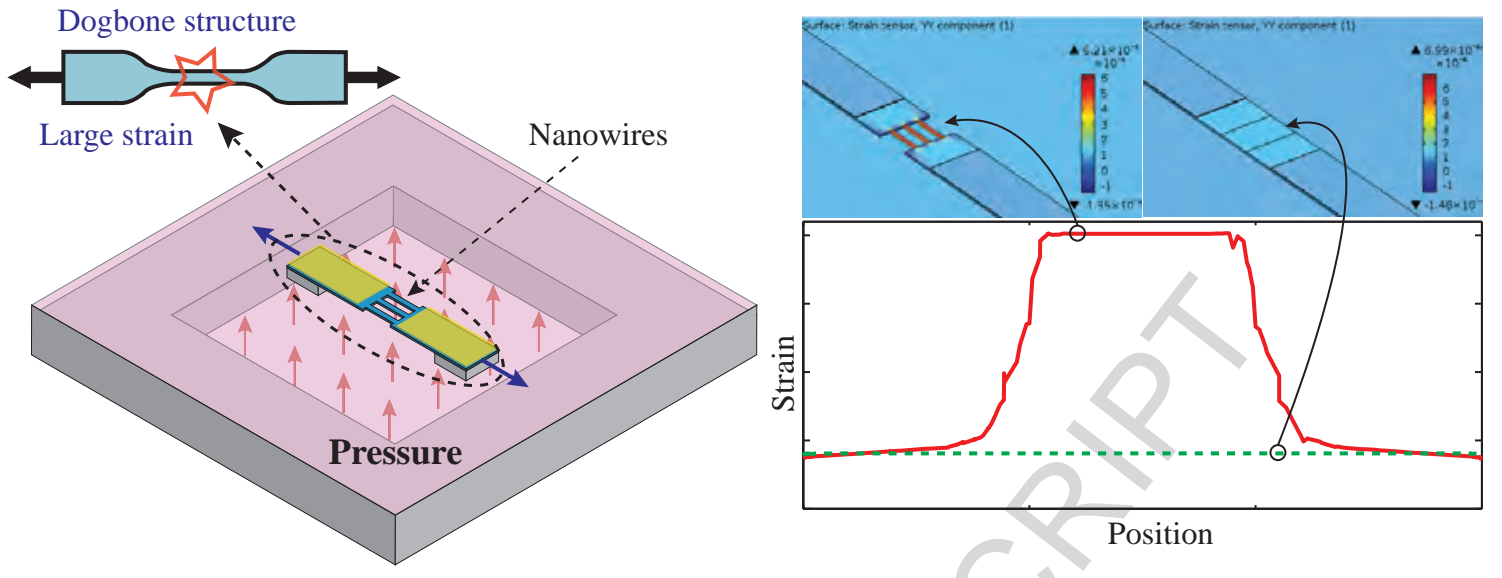


\section{Highlights}

- A smart mechanical design employing the stress concentration phenomenon in nanowires was proposed to enhance the sensitivity in piezoresistive sensors.

- A numerical analysis model was developed to estimate the amplification gain of the proposed nanowire structure.

- A proof-of-concept nanowire-pressure sensor was fabricated and characterized showing significant 3 -fold enhancement in the sensitivity in comparison to conventional structures 


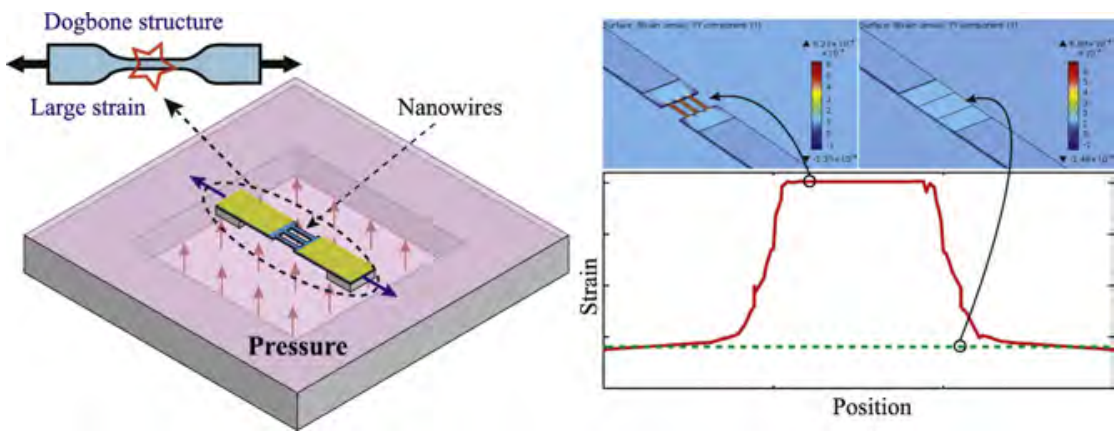

Graphics Abstract 


\section{(a) Concept of pressure sensors}

Dogbone structure

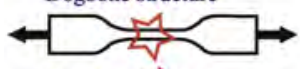

Large strain (b) Principle

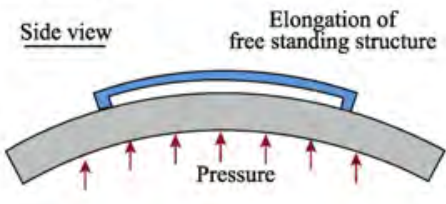

Top view
$+2$

Nanowires

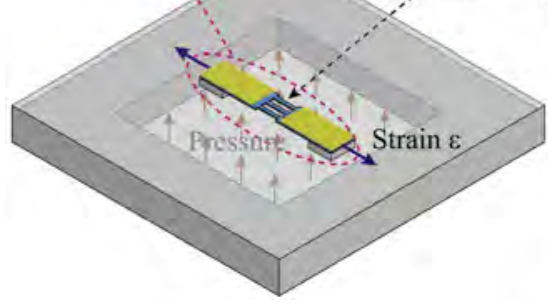

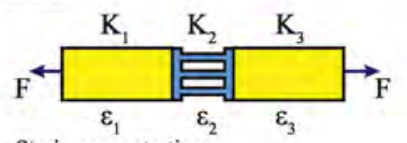

Strain concentration: $\varepsilon_{2} \gg \varepsilon_{1}$ (c) Numerical estimation

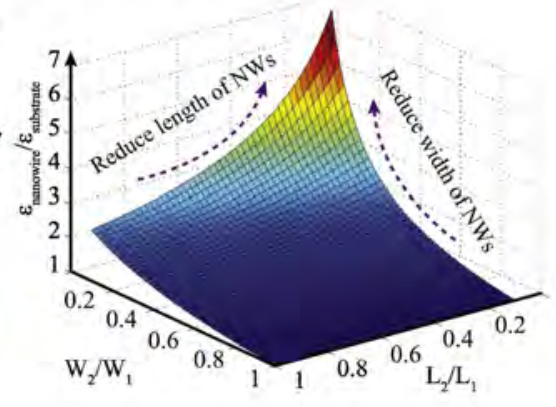

Figure 1 
(1)

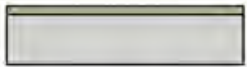

(3)

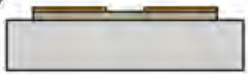

(5)

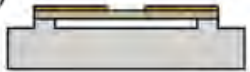

Al

(2)

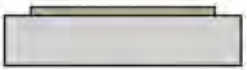

(4)

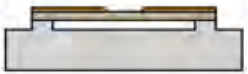

(6)

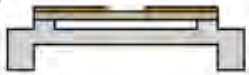

$\mathrm{SiC} \mathrm{Si}$

Figure 2 


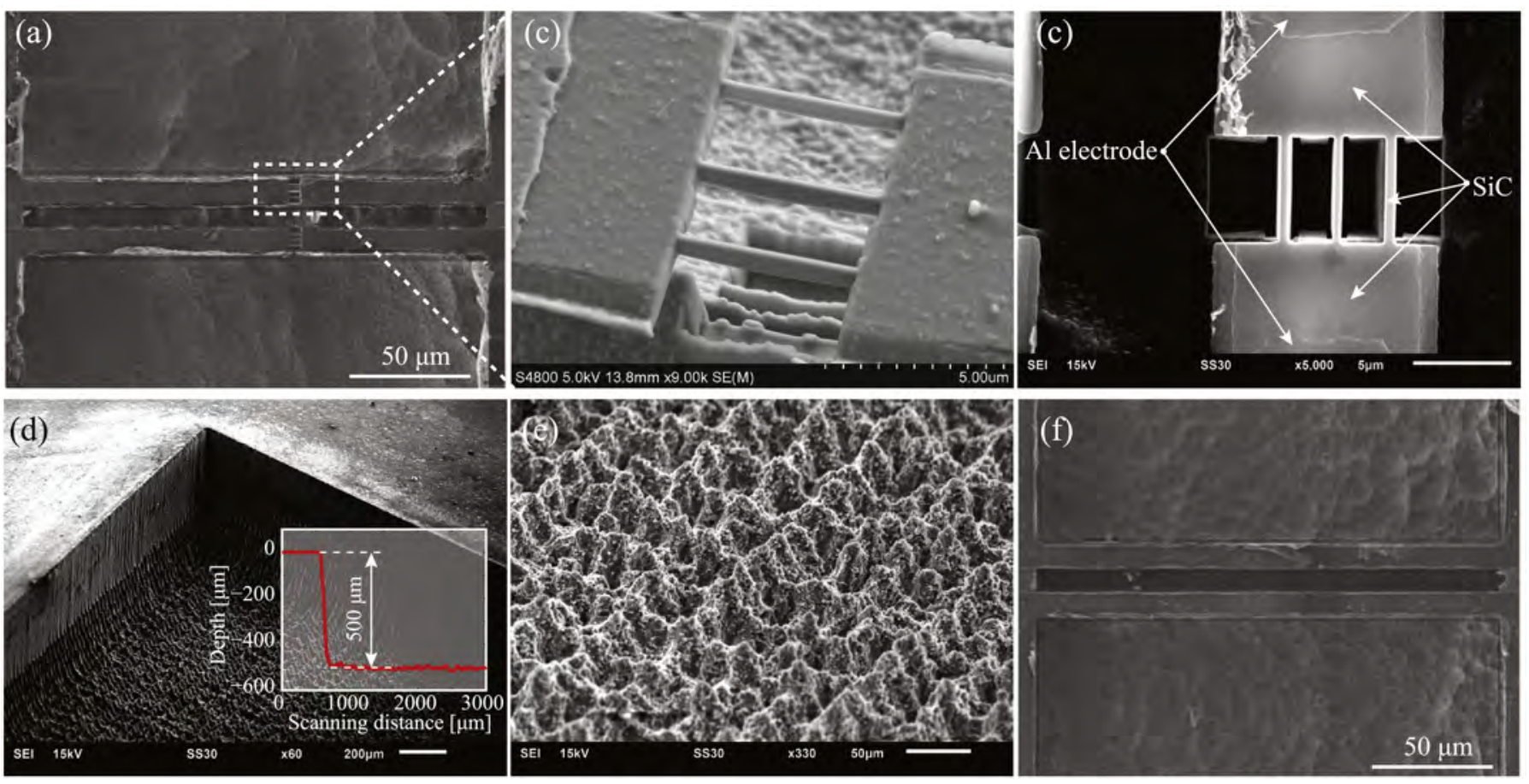

Figure 3 


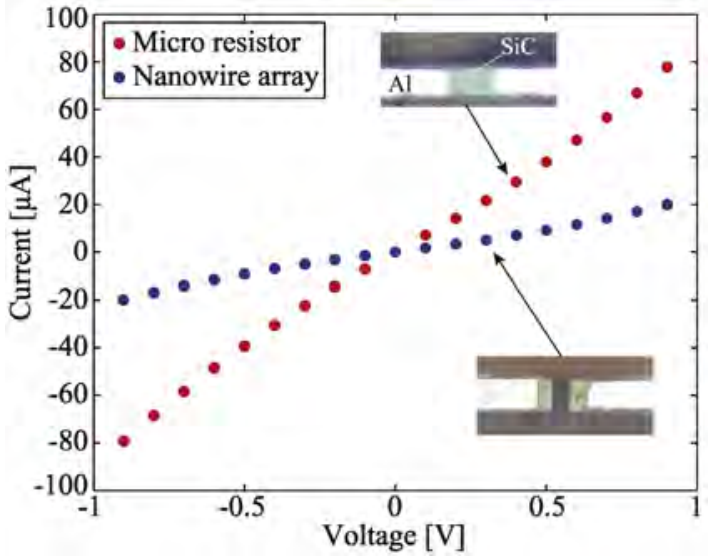

Figure 4 


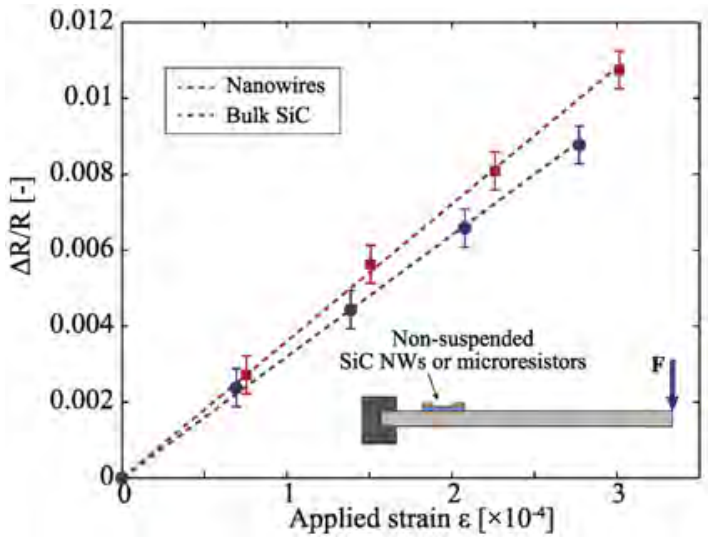

Figure 5 
(a)

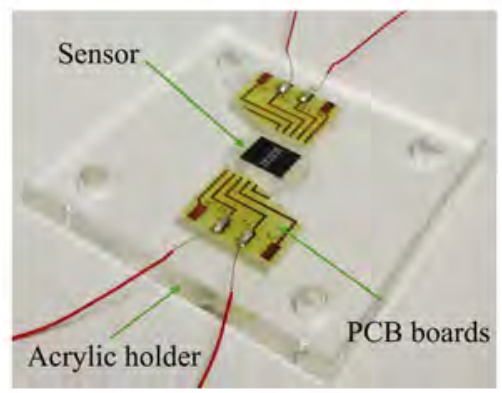

(d)

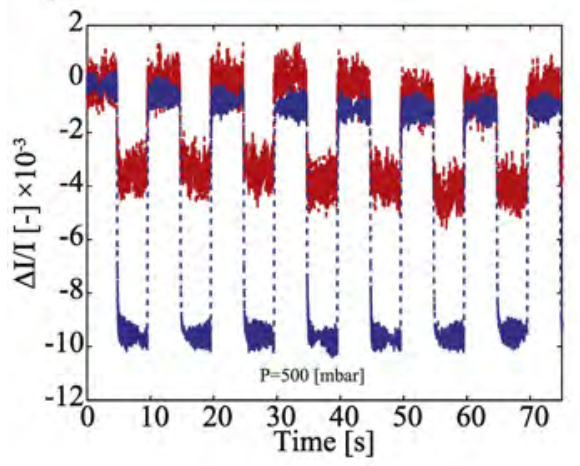

(b)

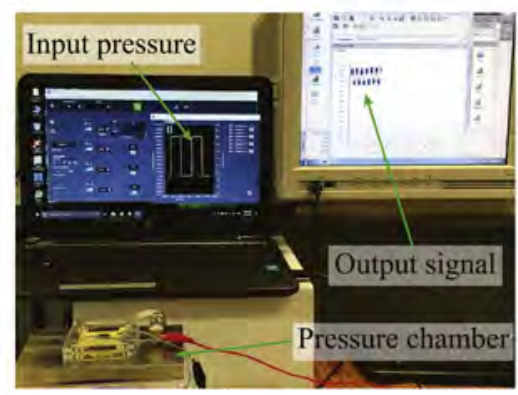

(e)

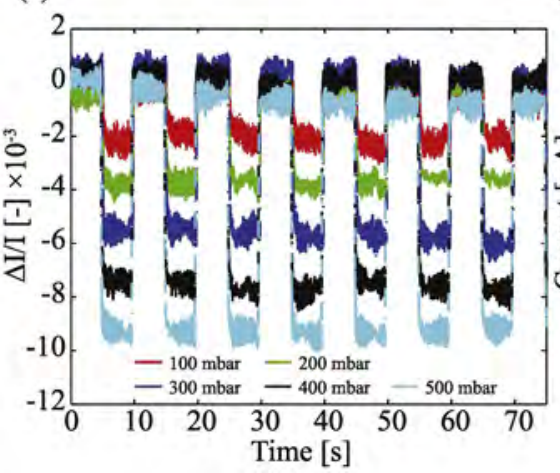

(c)

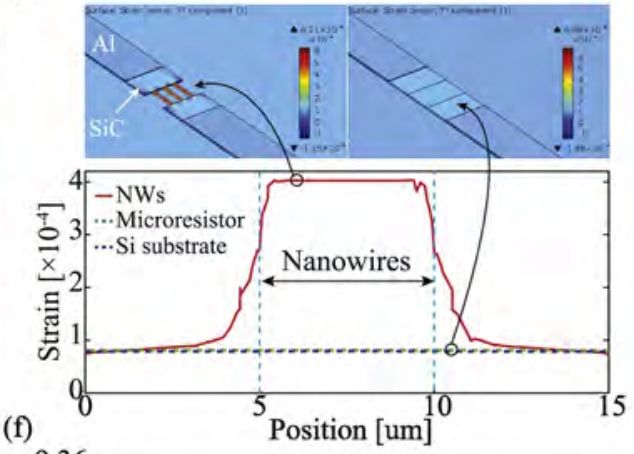

(1)

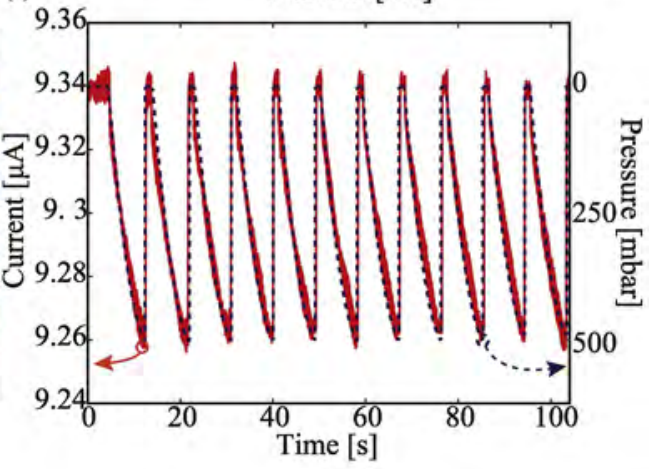

Figure 6 


\section{Accepted Manuscript}

Highly sensitive pressure sensors employing $3 \mathrm{C}-\mathrm{SiC}$ nanowires fabricated on a free standing structure

Hoang-Phuong Phan, Karen M. Dowling, Tuan Khoa Nguyen, Toan Dinh, Debbie G. Senesky, Takahiro Namazu, Dzung Viet Dao, Nam-Trung Nguyen

PII:

S0264-1275(18)30499-4

DOI: doi:10.1016/j.matdes.2018.06.031

Reference: JMADE 4001

To appear in: Materials \& Design

Received date:

3 April 2018

Revised date: 15 May 2018

Accepted date: 15 June 2018

Please cite this article as: Hoang-Phuong Phan, Karen M. Dowling, Tuan Khoa Nguyen, Toan Dinh, Debbie G. Senesky, Takahiro Namazu, Dzung Viet Dao, Nam-Trung Nguyen , Highly sensitive pressure sensors employing 3C-SiC nanowires fabricated on a free standing structure. Jmade (2018), doi:10.1016/j.matdes.2018.06.031

This is a PDF file of an unedited manuscript that has been accepted for publication. As a service to our customers we are providing this early version of the manuscript. The manuscript will undergo copyediting, typesetting, and review of the resulting proof before it is published in its final form. Please note that during the production process errors may be discovered which could affect the content, and all legal disclaimers that apply to the journal pertain. 